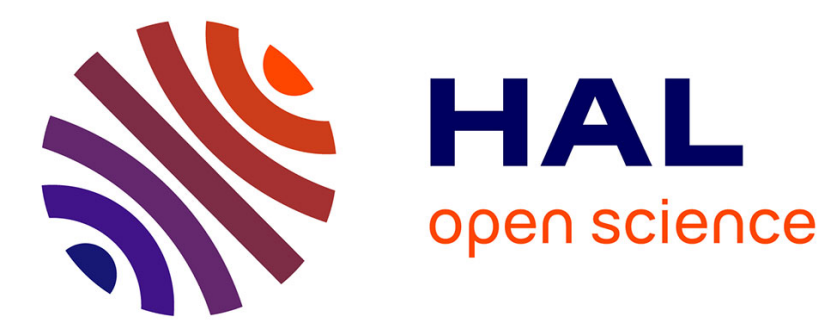

\title{
Étude d'un plasma d'argon fortement ionisé à basse pression produit par une décharge pulsée
}

J. Jolly

\section{To cite this version:}

J. Jolly. Étude d'un plasma d'argon fortement ionisé à basse pression produit par une décharge pulsée. Revue de Physique Appliquée, 1977, 12 (4), pp.553-558. 10.1051/rphysap:01977001204055300 . jpa00244209

\section{HAL Id: jpa-00244209 https://hal.science/jpa-00244209}

Submitted on 1 Jan 1977

HAL is a multi-disciplinary open access archive for the deposit and dissemination of scientific research documents, whether they are published or not. The documents may come from teaching and research institutions in France or abroad, or from public or private research centers.
L'archive ouverte pluridisciplinaire HAL, est destinée au dépôt et à la diffusion de documents scientifiques de niveau recherche, publiés ou non, émanant des établissements d'enseignement et de recherche français ou étrangers, des laboratoires publics ou privés. 


\title{
ÉTUDE D'UN PLASMA D'ARGON FORTEMENT IONISÉ A BASSE PRESSION PRODUIT PAR UNE DÉCHARGE PULSÉE
}

\author{
J. JOLLY \\ Laboratoire de Physique des Plasmas $\left({ }^{*}\right)$ Université Paris-Sud, \\ 91405 Orsay, France
}

(Reçu le 25 octobre 1976, révisé le 15 décembre 1976, accepté le 22 décembre 1976)

\begin{abstract}
Résumé. - On décrit une décharge électrique fonctionnant avec des impulsions de courant rectangulaires durant $25 \mu$ s et permettant d'atteindre des densités de courant supérieures à $1000 \mathrm{~A} / \mathrm{cm}^{2}$. On étudie le plasma obtenu dans l'argon, à des pressions de $5 \times 10^{-2}$ et $10^{-1}$ torr, au moyen de diagnostics spectroscopiques. La densité électronique, la température des ions et des neutres et la concentration des atomes métastables sont mesurées en fonction du courant de décharge. L'évolution de ces grandeurs pendant l'impulsion montre que le plasma fortement ionisé ainsi créé $\left(n_{\mathrm{e}} / n_{0} \simeq 1\right)$ est en régime quasi-stationnaire $5 \mu$ s après le début de l'impulsion.
\end{abstract}

\begin{abstract}
An electrical discharge in argon generated by a $25 \mu$ s rectangular current pulse of maximum current density higher than $1000 \mathrm{~A} / \mathrm{cm}^{2}$ is described. The argon plasma obtained in the pressure range $5 \times 10^{-2}, 10^{-1}$ torr is analysed spectroscopically. The electron density, the ion temperature, neutral temperature and metastable atom concentration are measured as a function of discharge current. The time resolved measurement of these quantities indicate that the highly ionized argon plasma $\left(n_{\mathrm{e}} / n_{0} \simeq 1\right)$ is quasi-stationary $5 \mu$ s after the beginning of the pulse.
\end{abstract}

1. Introduction. - Les décharges électriques fonctionnant en régime pulsé dans des tubes de faibles diamètres $(1 \mathrm{à} 10 \mathrm{~mm})$ permettent d'atteindre, avec une technologie relativement simple, des densités de courant très importantes. Outre leur intérêt pour la réalisation de lasers ioniques, ces décharges permettent d'obtenir au laboratoire des plasmas fortement ionisés et d'étudier les propriétés des ions excités et les transferts d'excitation ion-molécule. Les impulsions présentent par contre l'inconvénient de créer des plasmas en régime non stationnaire dans lesquels les effets transitoires rendent difficiles à la fois les mesures et leurs interprétations. Cependant un choix correct de la forme et de la longueur de l'impulsion permet de pallier ces difficultés.

Dans cet article, après avoir décrit le dispositif expérimental utilisé, nous présentons les résultats des mesures concernant quelques caractéristiques $\mathrm{du}$ plasma : densité électronique, température des ions et des neutres et concentration des atomes métastables. Les mesures effectuées conduisent à des valeurs moyennées sur le rayon du tube. La variation de ces grandeurs en fonction du courant de décharge et leurs évolutions dans le temps pendant l'impulsion permettent de conclure que le plasma produit est en régime

$\left(^{*}\right)$ Laboratoire associé au C. N. R.S. quasi-stationnaire pendant la majeure partie de l'impulsion.

2. Dispositif expérimental. - A basse pression et pour les fortes intensités de courant les décharges pulsées présentent des instabilités caractérisées par des oscillations de grande amplitude et haute fréquence de la tension aux bornes et des discontinuités dans le courant de décharge. Ces instabilités apparaissent au bout d'un temps d'autant plus court que le courant est élevé et que la pression est plus basse.

Plusieurs mécanismes peuvent être responsables de ces instabilités. Le plus important semble être une réduction de la densité du gaz neutre dans le plasma atteignant un seuil critique au-delà duquel se produisent. des oscillations de la charge d'espace similaires à celles rencontrées dans les diodes à plasma [1]. La réduction de la densité du gaz neutre, outre l'ionisation, peut avoir trois causes : le pompage du gaz par la décharge, le chauffage du gaz chassant les atomes à l'extérieur du tube et l'absorption du gaz sur les parois. L'effet de pompage peut être important pour des impulsions longues ayant un taux de répétition élevé [2]. Le chauffage du gaz ne peut être mis en cause que si pendant l'impulsion un grand nombre de particules a le temps de quitter le tube. L'absorption du gaz sur les parois peut avoir un effet important du fait du fort courant ionique radial dans les décharges à basse 
pression. Si on suppose que les ions tombant sur les parois restent absorbés pendant un certain temps avant de redonner un atome neutre, on peut obtenir une diminution sensible de la densité du gaz dans le plasma.

Pour éviter ces instabilités, rendant très difficile l'emploi d'appareils électroniques pour les mesures, nous avons choisi de travailler avec une impulsion dont la durée reste inférieure au temps d'apparition des instabilités dans la gamme de pression et de courant utilisé. $\mathrm{Au}$ cours d'études préliminaires nous avons employé des impulsions de diverses longueurs et de diverses formes ce qui nous a amené à choisir pour ce travail des impulsions de courant rectangulaires durant $25 \mu$ s et ayant une fréquence de répétition de $10 \mathrm{~Hz}$. La décharge se fait dans un tube en pyrex ou en quartz de diamètre intérieur $6 \mathrm{~mm}$ et de longueur 50 à $100 \mathrm{~cm}$. Pour diminuer les effets de pompage du gaz par la décharge on utilise un retour de gaz entre l'anode et la cathode ayant une longueur double de celle du tube. La cathode est constituée d'un cylindre en tantale de $4 \mathrm{~mm}$ de diamètre dans lequel on injecte du gaz afin de faciliter l'amorçage de la décharge, le flux d'argon qui y circule est pompé sans être entraîné dans le tube à décharge de sorte que les produits de pulvérisation de la cathode ne polluent pas la décharge. L'anode cylindrique en tantale est montée coaxialement au tube. Les impulsions sont produites par une ligne à constantes localisées $L C$ dont l'impédance est $Z=\sqrt{L / C}$ et la durée de l'impulsion $\tau=2 n \sqrt{L . C}$ où $L$ et $C$ sont la self et la capacité de chaque élément et $n$ le nombre d'éléments $L C$ constituant la ligne.

Si l'impédance du circuit de décharge est égale à l'impédance caractéristique de la ligne, l'impulsion est de forme rectangulaire et le temps de montée est sensiblement égal au temps caractéristique d'un élément. Le générateur utilisé dans cette étude est constitué de 12 éléments $L C$ avec $L=5 \mu \mathrm{H}$ et $C=0,2 \mu \mathrm{F}$. Le circuit électrique est fermé par le tube qui joue luimême le rôle d'interrupteur. L'impulsion est déclenchée en appliquant sur un fil bobiné autour du tube une impulsion haute tension $(30 \mathrm{kV}, 0,5 \mu \mathrm{s})$ préionisant le gaz. La forme et l'intensité du courant de décharge sont enregistrées aux bornes d'un shunt de Park de résistance $5 \times 10^{-3} \Omega$. En variant la tension de charge de la ligne et la résistance $R_{\mathrm{D}}$ on obtient des impulsions de courant dont l'intensité est comprise entre 50 et $300 \mathrm{~A}$ ce qui correspond à des densités de courant de 175 à $1060 \mathrm{~A} / \mathrm{cm}^{2}$. Des densités de courant beaucoup plus importantes ont été obtenues $\left(I=850 \mathrm{~A}, j=3000 \mathrm{~A} / \mathrm{cm}^{2}\right)$ avec des taux de répétition plus faibles de telle façon que le courant moyen dans le tube reste inférieur à $100 \mathrm{~mA}$.

On présente figure 1 le dispositif expérimental décrit ci-dessus ainsi que les systèmes de mesure utilisés dans la suite de ce travail et figure 2 un oscillogramme du courant pour $I=300 \mathrm{~A}$.



Fig. 1. - Représentation schématique du dispositif expérimental.

[Schematic of the experimental arrangement.]

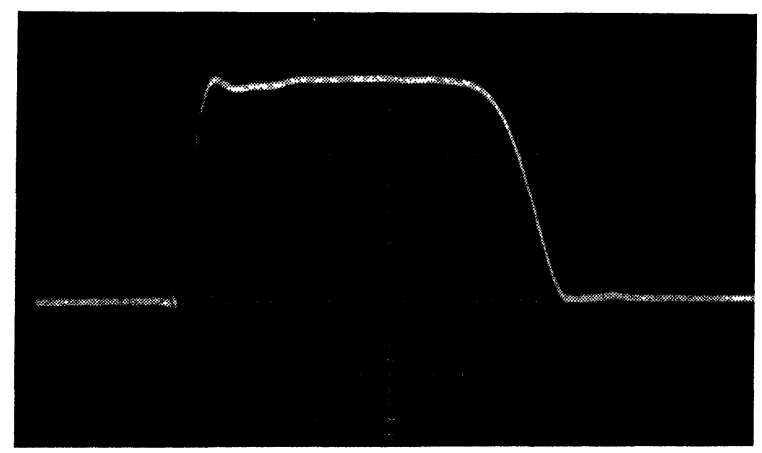

Fig. 2. - Oscillogramme du courant. Verticalement $100 \mathrm{~A} /$ div. Horizontalement $5 \mu \mathrm{s} / \mathrm{div}$.

[Oscilloscope trace of the current. Vertical scale: Discharge current $100 \mathrm{~A} / \mathrm{div}$. Horizontal scale : Time $5 \mu \mathrm{s} / \mathrm{div}$.]

3. Mesure de la température des ions et des neutres. - La valeur de la température des ions et des neutres intervient dans cette étude à plusieurs reprises. Elle est nécessaire pour déterminer la densité électronique du plasma et pour mesurer la population des niveaux excités de l'atome et de l'ion.

Nous avons calculé les températures $T_{\mathrm{i}}$ et $T_{0}$ à partir de la largeur Doppler des raies émises dans l'argon neutre et ionisé d'après la formule :

$$
\Delta v_{\mathrm{D}}=2 \sqrt{2 R \operatorname{Ln} 2} \frac{v_{0}}{C} \sqrt{\frac{T}{M}} .
$$


$R$ est la constante des gaz parfaits, $v_{0}$ la fréquence centrale de la raie étudiée, $C$ la vitesse de la lumière et $M$ la masse molaire du gaz.

Les profils des raies sont analysés au moyen d'un interferomètre Fabry-Pérot dont le réglage et le balayage se font au moyen de céramiques piezoélectriques. Pour une radiation incidente ayant un certain profil, l'intensité transmise $I_{\mathrm{T}}$ est donnée par la convolution de la largeur d'appareil et du profil de la raie. Ballik [3] a déterminé la forme fonctionnelle de $I_{\mathrm{T}}$ pour une radiation incidente ayant un profil de Voigt et une fonction instrumentale donnée par l'équation d'Airy.

$$
I_{\mathrm{T}}=\frac{\frac{1}{2}+\sum_{n=1}^{\infty}\left(R^{\prime} \mathrm{e}^{-L}\right)^{n} \mathrm{e}^{-n^{2} D^{2} / 4} \cos n z}{\frac{1}{2}+\sum_{m=1}^{\infty}\left(R^{\prime} \mathrm{e}^{-L}\right)^{m} \mathrm{e}^{-m^{2} D^{2} / 4}}
$$

avec

$$
z=2 \pi \frac{v_{0}-v}{\Delta v_{\mathrm{i}}}
$$

$\Delta v_{\mathrm{i}}$ étant l'intervalle spectrale libre entre deux pics de transmission du Fabry-Pérot. Les paramètres $D$ et $R^{\prime} \mathrm{e}^{-L}$ sont suffisants pour déterminer la forme de $I_{\mathrm{T}}$

$$
D=\pi \frac{\Delta v_{\mathrm{D}}}{\Delta v_{\mathrm{i}} \sqrt{\operatorname{Ln} 2}}, \quad L=\pi \frac{\Delta v_{\mathrm{L}}}{\Delta v_{\mathrm{i}}} .
$$

$\Delta v_{\mathrm{D}}$ et $\Delta v_{\mathrm{L}}$ étant les largeurs à mi-hauteur des profils des composantes Gaussienne et Lorentzienne. $R^{\prime}$ est la valeur effective de la réflectivité des miroirs tenant compte des effets limitant la résolution. $R^{\prime}$ étant très difficile à évaluer, la méthode de Ballik ne permet pas d'extraire facilement la composante Lorentzienne de la raie étudiée par contre elle est idéale pour connaître la composante Gaussienne puisque la connaissance de la largeur instrumentale n'est pas nécessaire. Le paramètre $D$ est déterminé en utilisant la méthode de résolution graphique de Ballik à partir des courbes expérimentales de la transmission du Fabry-Pérot pour une radiation donnée. Pour mesurer la température des neutres nous avons utilisé la transition $2 \mathrm{p}^{2}-1 \mathrm{~s}^{5} \quad(\lambda=6965 \AA)$ et pour les ions $4 \mathrm{p}^{2} D_{5 / 2}^{0}-4 \mathrm{~s}^{2} \mathrm{P}_{3 / 2}(\lambda=4880 \AA)$. Ces raies sont isolées au moyen de filtres interférentiels de bande passante $\Delta \lambda=10 \AA$. Les mesures sont faites selon l'axe du tube. $\mathrm{La}$ résolution dans le temps des mesures est obtenue au moyen d'un échantillonneur-moyenneur (Boxcar) utilisant des échantillons de durée $0,5 \mu \mathrm{s}$ prélevés au bout d'un temps déterminé après le départ de l'impulsion et moyennés sur un grand nombre d'impulsions.

L'évolution de la température des atomes et des ions, pendant l'impulsion, a été mesurée dans la portion plane de l'impulsion de courant entre 5 et $18 \mu \mathrm{s}$. La température des ions, dans cet intervalle de temps,

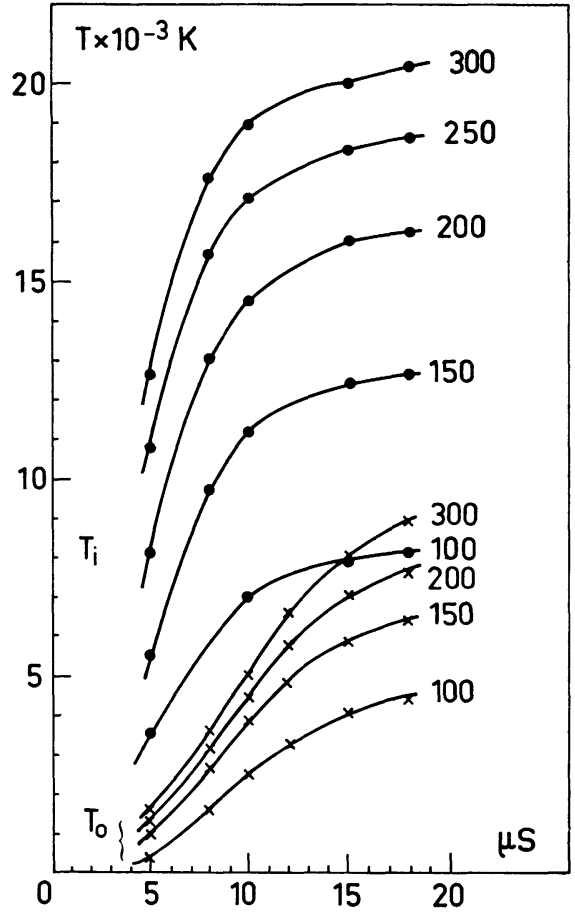

FIG. 3. - Température des ions $(\bullet)$ et des neutres $(X)$ pendant l'impulsion. Pour chaque courbe figure le courant de décharge en ampères. Pression de remplissage $5 \times 10^{-2}$ torr.

[Time dependences of ion $(\bullet)$ and neutral $(x)$ temperatures for various discharge currents given in amperes. Filling pressure : $5 \times 10^{-2}$ torr.]



Fig. 4. - Température des ions et des neutres, mesurée $15 \mu \mathrm{s}$ après le début de l'impulsion, en fonction du courant de décharge.

Pression de remplissage $(\times) 10^{-1}$ torr, (O) $5 \times 10^{-2}$ torr.

[Current dependences of ion and neutral temperatures measured $15 \mu$ s after the start of the pulse. Filling pressure : $(X) 10^{-1}$ torr, (๑) $5 \times 10^{-2}$ torr.] 
croît d'un facteur de l'ordre de 2 , d'abord très rapidement puis tend vers une température limite. La température des neutres augmente tout au long de l'impulsion. Les résultats sont présentés figure 3 . Les variations de $T_{\mathrm{i}}$ et $T_{0}$, en fonction du courant de décharge, mesurées $15 \mu$ s après le début de l'impulsion sont présentées figure 4 . Les températures $T_{\mathrm{i}}$ et $T_{0}$ varient peu pour un courant donné dans la gamme de pression étudiée $\left(2 \times 10^{-2}-10^{-1}\right.$ torr $)$ les différences observées restent inférieures aux incertitudes expérimentales figurées par les barres d'erreurs.

4. Mesure de la densité électronique. - On détermine la densité électronique à partir de la mesure de l'élargissement par effet Stark linéaire de la raie $\mathrm{H}_{\beta}$ de l'hydrogène [4]. La pression d'hydrogène ajoutée dans la décharge est de $1 \%$ de la pression d'argon. Le profil de la raie $\mathrm{H}_{\beta}$ est observé au moyen d'un monochromateur à haute résolution $(\lambda / \Delta \lambda \simeq 30000)$. Le profil obtenu résulte de la superposition d'un profil gaussien dû à l'effet Doppler et du profil Stark de $\mathbf{H}_{\boldsymbol{\beta}}$. Pour extraire la largeur Stark de la largeur mesurée il faut connaître la largeur Doppler, c'est-à-dire la température des atomes d'hydrogène. Il est très difficile de déterminer cette température, cependant en écrivant les taux de transfert d'énergie entre gaz électronique, gaz ionique et gaz atomique on peut montrer que ce sont les transferts ion-atome avec échange de charge qui sont responsables du chauffage du gaz atomique. Le transfert $\mathrm{Ar}^{+}-\mathrm{H}$ n'étant pas symétrique est moins efficace que le transfert $\mathrm{Ar}^{+}-\mathrm{Ar}$. On peut donc supposer que la température des atomes d'hydrogène est inférieure ou au plus égale à celle des atomes d'argon. Le tableau I donne, en fonction du courant

\section{TABLEAU I}

Pression de remplissage $5 \times 10^{-2}$ torr

\begin{tabular}{cccc}
$\begin{array}{c}\text { Courant } \\
\text { de décharge } \\
I(\mathrm{~A})\end{array}$ & $\begin{array}{c}\text { Largeur } \\
\text { mesuré } \\
\Delta \lambda(\AA)\end{array}$ & $\begin{array}{c}\text { Largeur } \\
\text { Doppler } \\
\Delta \lambda_{\mathbf{D}}(\AA)\end{array}$ & $\begin{array}{c}\text { Largeur } \\
\text { Stark } \\
\Delta \lambda_{\mathbf{S}}(\AA)\end{array}$ \\
\hline 50 & - & - & - \\
100 & 0,66 & 0,15 & 0,62 \\
150 & 1,1 & 0,22 & 1,05 \\
200 & 1,47 & 0,26 & 1,42 \\
250 & 1,75 & 0,29 & 1,70 \\
300 & 1,9 & 0,30 & 1,84 \\
& 2 & 0,31 & 1,95
\end{tabular}

de décharge, les largeurs à mi-hauteur mesurées $\Delta \lambda$, les largeurs Doppler $\Delta \lambda_{\mathrm{D}}$ calculées en supposant que les atomes d'hydrogène sont à la même température que les atomes d'argon et $\Delta \lambda_{\mathrm{s}}$ les largeurs Stark correspondantes. On a approximé le profil Stark à un profil lorentzien pour faciliter l'extraction de $\Delta \lambda_{\mathrm{s}}$ à partir d'un profil de Voigt en utilisant les tables de Davies et Vaughan [5].
La densité électronique est calculée à partir de la formule de Griem [6]

$$
n_{\mathrm{e}}=C\left(n_{\mathrm{e}}, T_{\mathrm{e}}\right)\left(\Delta \lambda_{\mathrm{L}}\right)^{3 / 2} .
$$

Le coefficient $C\left(n_{\mathrm{e}}, T_{\mathrm{e}}\right)$ varie rapidement en fonction de $n_{\mathrm{e}}$ et très lentement en fonction de $T_{\mathrm{e}}$ (entre $10^{4}$ et $4 \times 10^{4} \mathrm{~K} C\left(n_{\mathrm{e}}, T_{\mathrm{e}}\right)$ varie de moins de $\left.2 \%\right)$. Les résultats présentés figure 5 font apparaître une variation linéaire de $n_{\mathrm{e}}$ en fonction du courant de décharge

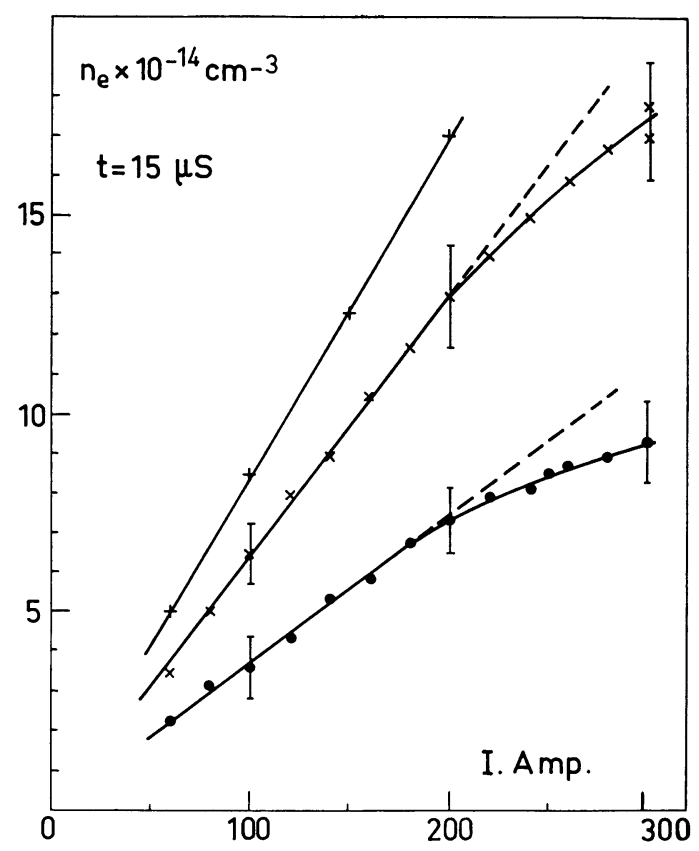

Fig. 5. - Densité électronique en fonction du courant de décharge mesurée au temps $t=15 \mu \mathrm{s}$. Pression de remplissage (+) $2 \times 10^{-1}$ torr, $(\times) 10^{-1}$ torr, $(\odot) 5 \times 10^{-2}$ torr.

[Current dependence of the electron concentration measured at the time $t=15 \mu$ s. Filling pressure : $(+) 2 \times 10^{-1}$ torr, $(\times)$ $10^{-1}$ torr, (๑) $5 \times 10^{-2}$ torr.]

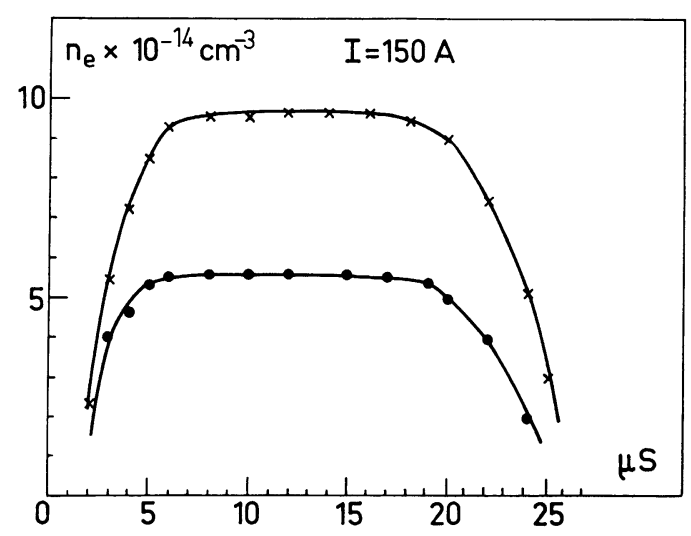

Fig. 6. - Densité électronique pendant l'impulsion pour un courant de décharge $I=150$ A. Pression de remplissage $(\times)$ $10^{-1}$ torr, (O) $5 \times 10^{-2}$ torr.

[Time dependence of the electron concentration for a discharge current of 150 Amperes. Filling pressure : $(\times) 10^{-1}$ torr, (O) $5 \times 10^{-2}$ torr.] 
pour les faibles valeurs de $I$. Pour les intensités plus élevées une saturation apparaît due à la raréfaction du gaz neutre. On a également mesuré l'évolution de la densité électronique en fonction du temps pendant l'impulsion. Les résultats sont présentés figure 6 .

5. Mesure de la densité des atomes métastables. Nous avons montré dans une étude précédente que les états métastables du niveau $4 \mathrm{~s}$ jouent un rôle important dans les mécanismes d'ionisation [7]. Il est donc intéressant de connaître la population des niveaux métastables ainsi que leur évolution dans le temps pendant l'impulsion. Pour mesurer la concentration des atomes métastables nous avons utilisé une méthode d'auto-absorption permettant de déterminer le coefficient d'absorption du milieu à partir de la mesure du rapport des intensités totales de deux raies partiellement autoabsorbées aboutissant sur le niveau dont on veut déterminer la population. Cette méthode a été présentée dans une précédente publication [8].

La densité des atomes métastables $n_{\mathrm{M}}$ s'obtient à partir du coefficient d'absorption $k_{0}$ et de la température des neutres $T_{0}$ d'après la formule

$$
n_{\mathrm{M}}=1,375 \times 10^{5} \frac{k_{0}}{f \lambda}\left(T_{0}\right)^{1 / 2},
$$

où $f$ et $\lambda$ sont la force d'oscillateur et la longueur d'onde de la transition utilisée.

Les résultats sont présentés figures 7 et 8 respectivement en fonction du courant de décharge $15 \mu \mathrm{s}$ après le début de l'impulsion et en fonction du temps pendant l'impulsion pour $I=150 \mathrm{~A}$. Les concentrations de métastables obtenus pour des pressions de remplissage de $5 \times 10^{-2}$ et $10^{-1}$ torr sont sensiblement identiques compte tenu des erreurs expérimentales.

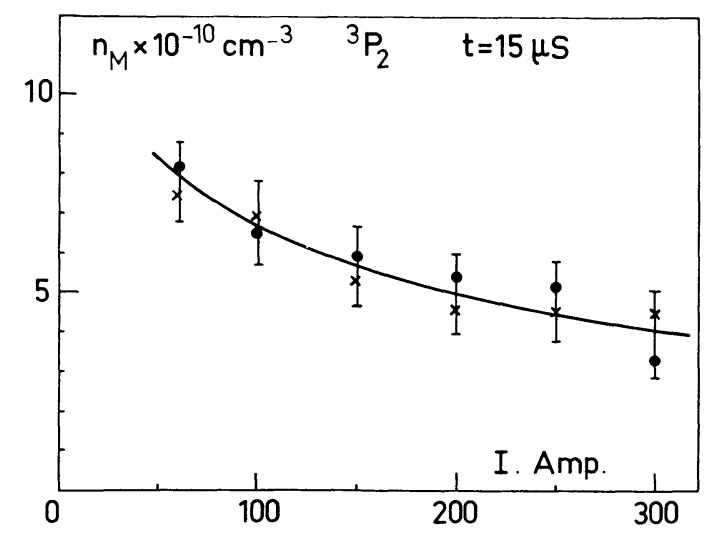

Fig. 7. - Densité des métastables du niveau ${ }^{3} P_{2}$ en fonction du courant de décharge mesurée au temps $t=15 \mu \mathrm{s}$. Pression de remplissage $(X) 10^{-1}$ torr, $(0) 5 \times 10^{-2}$ torr.

[Current dependence of the neutral metastable concentration ${ }^{3} \mathrm{P}_{2}$ at the time $t=15 \mu \mathrm{s}$. Filling pressure : $(\times) 10^{-1}$ torr, (ब) $5 \times 10^{-2}$ torr.]

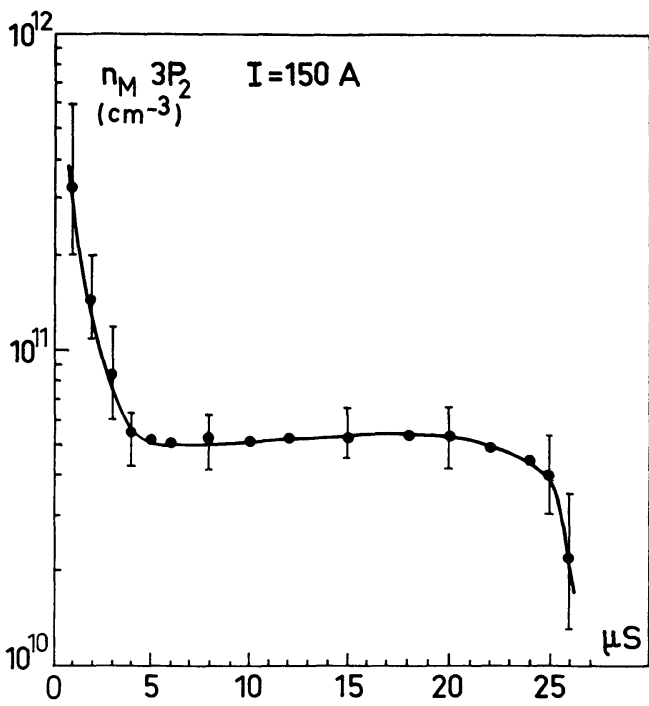

FIg. 8. - Densité des métastables du niveau ${ }^{3} \mathbf{P}_{2}$ pendant l'impulsion pour un courant $I=150 \mathrm{~A}$. Pression de remplissage $5 \times 10^{-2}$ torr.

[Time dependence of the neutral metastable concentration ${ }_{3} \mathrm{P}_{2}$ for a discharge current of 150 A. Filling pressure : $5 \times 10^{-2}$ torr.]

6. Conclusion. - Le choix de la longueur de l'impulsion a été fait d'une part dans le but de s'affranchir des instabilités discutées au paragraphe 2 et d'autre part afin d'éviter une diminution importante de la densité des particules lourdes due au chauffage du gaz. En supposant une vitesse thermique de l'ordre de $2 \times 10^{5} \mathrm{~cm} / \mathrm{s}$ seules les particules se trouvant à moins de $2 \mathrm{~cm}$ des extrémités peuvent avoir une chance de quitter le tube en $10 \mu$ s. On peut donc avec une bonne approximation écrire que la densité de gaz se conserve pendant l'impulsion c'est-à-dire que $n_{\mathrm{g}}=n_{0}+n_{\mathrm{i}}, n_{\mathrm{g}}$ étant la densité de gaz correspondant à la pression de remplissage et $n_{0}$ et $n_{\mathrm{i}}$ les concentrations d'atomes neutres et d'ions pendant l'impulsion. L'observation des résultats concernant l'évolution dans le temps de la densité des électrons et des métastables montre qu'après un intervalle de temps de l'ordre de $5 \mu$ s les concentrations ne changent que très peu. L'observation de la lumière émise par les niveaux excités aussi bien atomiques que ioniques conduit aux mêmes conclusions. On a donc pour la population de tous les niveaux $\mathrm{d} n_{\mathrm{j}} / \mathrm{d} t \simeq 0$ pour $t>5 \mu \mathrm{s}$.

La densité de population du niveau métastable peut s'écrire dans les conditions de saturation électronique

$$
n_{\mathrm{M}}=n_{0} \frac{C_{\mathrm{M}}\left(T_{\mathrm{e}}\right)}{D_{\mathrm{M}}\left(T_{\mathrm{e}}\right)},
$$

où $C_{\mathrm{M}}\left(T_{\mathrm{e}}\right)$ et $D_{\mathrm{M}}\left(T_{\mathrm{e}}\right)$ sont respectivement les coefficients de création et de destruction du niveau. Ces deux coefficients étant des fonctions croissantes de la température électronique, dans la gamme de température qui nous concerne $\left(k T_{\mathrm{e}} \leqslant 10 \mathrm{eV}\right)$, la quasistationnarité de $n_{\mathrm{M}}$ pendant l'impulsion entraîne celle 


\section{TABLEAU II}

$I=200 \mathrm{~A}$

$t=15 \mu \mathrm{s}$

$j\left(\mathrm{~A} / \mathrm{cm}^{2}\right)$

$5 \times 10^{-2}$ torr

$n_{\mathrm{g}}\left(\mathrm{cm}^{-3}\right)$

$1,45 \times 10^{15}$

$n_{\mathrm{o}}\left(\mathrm{cm}^{-3}\right)$

$7,1 \times 10^{14}$

$n_{\mathrm{e}}\left(\mathrm{cm}^{-3}\right)$

$n_{\mathrm{e}} / n_{\mathrm{o}}$

$7,4 \times 10^{14}$

$n_{\mathrm{M}}\left(\mathrm{cm}^{-3}\right)$

$T_{\mathrm{o}}(\mathrm{K})$

$T_{\mathrm{i}}(\mathrm{K})$

$T_{\mathrm{e}}(\mathrm{K})$
707

1

Pression de remplissage

$10^{-1}$ torr

707

$2,9 \times 10^{15}$

$1,6 \times 10^{15}$

$1,3 \times 10^{15}$

0,8

$5 \times 10^{10}$

7800

16700

35000 de la température électronique. Le plasma créé est donc en régime quasi continu entre 5 et $18 \mu$ s après le début de l'impulsion. Nous résumons dans le tableau II les grandeurs caractéristiques du plasma mesurées au temps $t=15 \mu$ s pour un courant de décharge de $200 \mathrm{~A}$. La densité de remplissage $n_{\mathrm{g}}$ est calculée à partir de la pression de remplissage et de la température du tube en fonctionnement, mesuré avec un thermocouple, en supposant que le gaz se met en équilibre avec les parois entre deux impulsions. Les valeurs de la température électronique données à titre indicatif dans le tableau II ont été calculées d'après un modèle théorique de la colonne positive aux fortes densités de courant qui sera publié ultérieurement.

Remerciements. - Nous remercions le Professeur J. L. Delcroix pour ses encouragements et ses suggestions à la lecture du manuscrit.

\section{Bibliographie}

[1] Tuma, D., Ware, A., Phys. Fluids 11 (1968) 1206.

[2] Neusel, R., IEEE J. Quant. Electron. QE-2 (1966) 331.

[3] Ballik, F., Appl. Opt. 5 (1966) 170.

[4] Wirdman, D., Quartely Progress Report No 107. Research Laboratory of Electronics M. I. T. (1972) 73.

[5] Davies, J. T., Vaughan, J. M., Astrophys. J. 137 (1963) 1302.
[6] Griem, H. R., Plasma Spectroscopy (Mc Graw-Hill Book Comp.) 1964, p. 170.

Kepple, P., Griem, H. R., Phys. Rev. 173 (1968) 317

[7] Jolly, J., Rapport L. P. 160 (1976) Laboratoire de Physique des Plasmas, 91405 Orsay.

[8] Jolly, J., Touzeau, M., J. Quant. Spectros. Radiat. Transfer. 15 (1975) 863. 